This item was submitted to Loughborough's Research Repository by the author.

Items in Figshare are protected by copyright, with all rights reserved, unless otherwise indicated.

\title{
Detection of terrorist threats in air passenger luggage: expertise
} development

\section{PLEASE CITE THE PUBLISHED VERSION}

\section{PUBLISHER}

(C) IEEE

\section{VERSION}

VoR (Version of Record)

\section{LICENCE}

CC BY-NC-ND 4.0

\section{REPOSITORY RECORD}

Liu, Xi, Alastair G. Gale, and Tao Song. 2019. "Detection of Terrorist Threats in Air Passenger Luggage: Expertise Development". figshare. https://hdl.handle.net/2134/6266. 
This item was submitted to Loughborough's Institutional Repository (https://dspace.lboro.ac.uk/) by the author and is made available under the following Creative Commons Licence conditions.

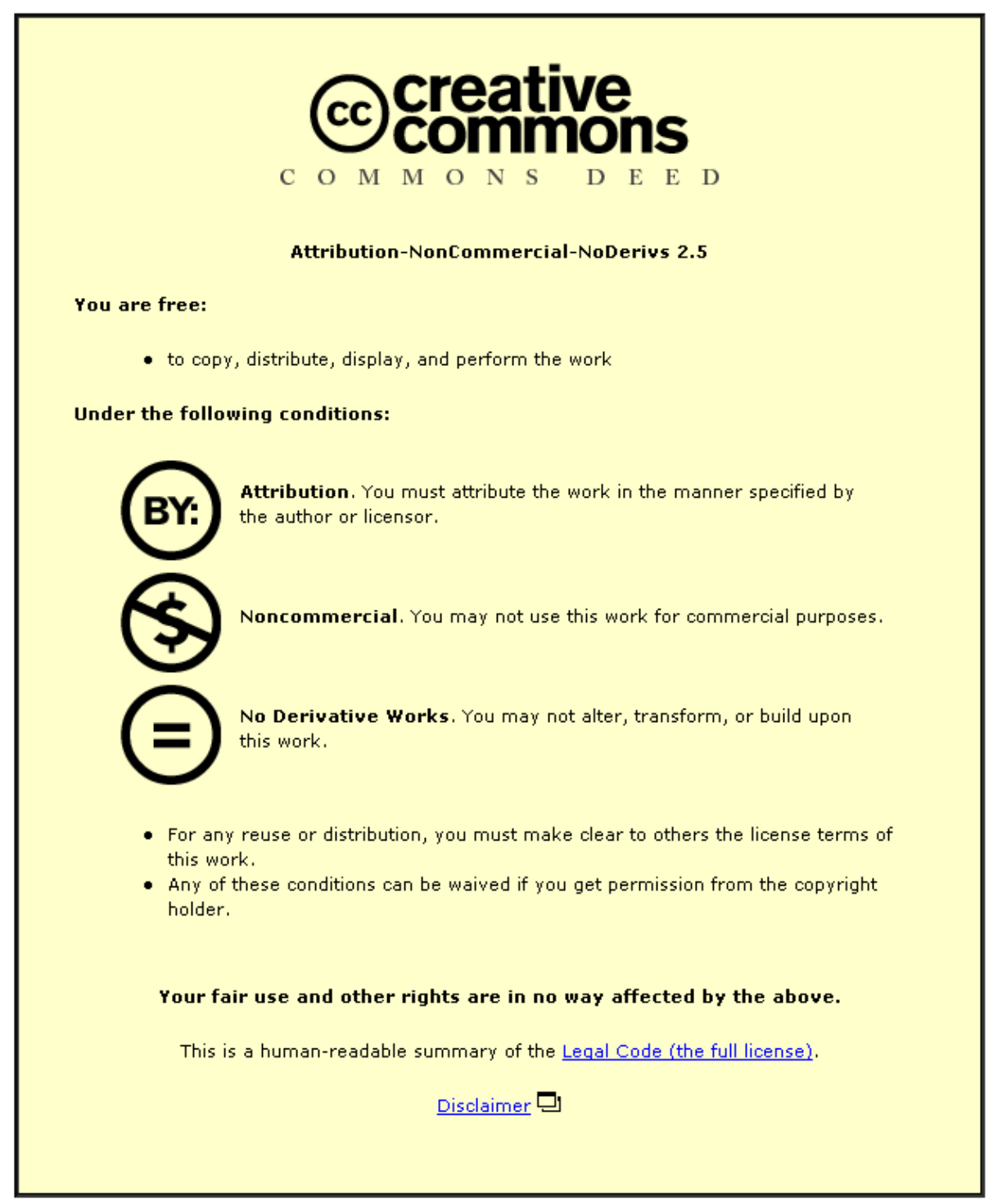

For the full text of this licence, please go to: http://creativecommons.org/licenses/by-nc-nd/2.5/ 


\section{Detection of Terrorist Threats in Air Passenger Luggage: Expertise Development}

\author{
Xi Liu \\ Applied Vision Research Centre \\ Loughborough University \\ Loughborough \\ LE11 3UZ \\ UK
}

\author{
Alastair Gale \\ Applied Vision Research Centre \\ Loughborough University \\ Loughborough \\ LE11 3UZ \\ UK
}

\author{
Tao Song \\ Applied Vision Research Centre \\ Loughborough University \\ Loughborough \\ LE11 3UZ \\ UK
}

\begin{abstract}
Currently, detecting potential threats in air passenger baggage heavily depends on the human examination of $X$-ray images of individual luggage items. In order to improve the performance of airport security personnel in searching images of air passenger luggage it is important first to understand fully the requirements of the demanding task. Here, an experiment is reported where eye movements of naïve observers and screeners were recorded when they searched $30 \mathrm{X}$-ray images of air passenger luggage for potential terrorist threat items such as guns, knives and improvised explosive devices. Compared with novices, the advantages of the screeners were speed and accuracy in detecting threats. Eye position data revealed that screeners were faster to fixate on target areas and once they fixated on targets their hit rate was significantly higher. Most of the IEDs were missed by both naîve observers and screeners due to interpretation errors which indicated the importance of training. Stimulus salience at the first fixation locations of naïve observers and screeners was compared to investigate expertise development. It was found that experience did not change attention preference on stimuli properties at the beginning of the observers visual search. The implications and further studies are discussed.
\end{abstract}

Index Terms - passenger luggage, visual search, expertise, salience.

\section{INTRODUCTION}

Air passenger luggage inspection within aviation security has become an important topic of how to keep explosives and other potential threats off aircraft. Although the American Transportation Security Administration (TSA) contemplates using computed tomography (CT) technology for screening carry-on bags, the technology for luggage examination has not changed much, as CT is more expensive than current $X$ ray systems, and for other reasons. The detection and recognition of potential threat items heavily depends on human interpretation of $\mathrm{X}$-ray luggage images. The most useful information provided by traditional one-view $X$-ray technology and expressed on a luggage image is an object's density which loses the texture and the colour of the object, and can make the object's edge and shape indistinct. Unfortunately, from 'shoe-bombs' to the recent UK liquid explosives plot, terrorist threats are both productive and diverse. Screeners work under high levels of noisy and timepressurized environments and search for weak, infrequent and changeable targets in two-dimensional X-ray images; so it is arduous for security screeners to maintain a high level of vigilance and knowledge about how to detect recurrent 'novel' threat targets among cluttered backgrounds. It is a challenging task for human limitations of perceptual and cognitive flexibility [1]. As a consequence, other than designing appropriate threat detection systems, it is essential to understand the requirements and extract task expertise so as to improve screener's capabilities.

In domains of medical images examination, and other realworld search, a two-stage visual search model has been developed which provides knowledge to aid understanding the task of airport security screeners [2-4]. In the first step, called the global stage, a pre-attentive filter is proposed to select a subset of features in a particular pattern for observer's attention and specific purposes. 'Pop-out' happens on the basis of simple local properties and for other factors, e.g., size, brightness and contrast. Then in the second stage of focal attention, every selected feature would be scrutinized and interpreted by the cognitive system to decide if it is a normal or abnormal area. This model assumes that the two stages are serial processes where the output of the first stage is the input of the second stage. We move our eyes to acquire visual information and alternate between very fast eye movement (saccade) and relatively stable gaze (fixation). Information is obtained and the location of the next fixation determined during the previous fixation and a saccade then executes to that location. The experimental evidence suggests that only part of the visual information available to the visual system is processed in full detail while the remainder is largely ignored [5]. What locations or features are selected in a search task? Alternatively, what determines the selected locations in the visual field? 
Some researchers accept that the first global stage is a rapid and bottom-up procedure, and the second focal stage is a slower phase with both bottom-up and top-down processes $[3,6]$. An important characteristic of experts in the domain of radiology is the speed and accuracy with which they complete the task [7-9]. It is argued that experts know both 'where' and 'what' to search for in their inspection of the radiological image because such experts apply skills in the areas of visual search, object recognition and decision making which are obtained from massive perceptual learning experience in their domain [9]. Experts generate representations of targets in their cognitive schema to test each 'candidate' in the visual field. Where the eyes move is determined by both cognitive representation and perceptual input which picks up visual information of potential targets. It seems that cognitive factors, including top-down mechanisms, such as task demands, both guides and interacts with the visual input in the selective attention activity of radiology. However, there is little direct evidence to verify this inference. If the top-down mechanism plays a more critical role in the task of airport security screening then a new solution based on semantic information of $X$-ray luggage images and task demands may be suggested for extracting expertise. Therefore, it is important to be clear about the procedure of visual selective attention in X-ray luggage image interpretation. Currently, the most popular and applicable computational visual attention model is Itti and Koch's pure bottom-up, saliency map model [10]. This ignores top-down control and cognitive factors. The saliency map approach provides a clear model which generates predictions for fixation locations and convenient computation for salience. It assumes at a given time the most salient location is localized, and the focus of attention should point to it. An "Inhibition of return" mechanism is introduced to avoid the attended location being examined again. A winner-takeall neural network is used to ensure only one location is selected every time. In previous work, the typical search of feature and conjunction search was reproduced by the model, where it had a similar search slope to human observers [11]. Search time is independent of the number of distractors in feature search while search time is nearly proportional to the number of distractors in conjunction search. Also the search performance of this model on complex natural scenes was faster than a human in $75 \%$ of the images. The model was also tested in a free viewing task and results indicated bottom-up mechanisms play a significant role in the allocation of attention [12]. However, visual saliency does not determine eye position during an active search task - counting the number of observers in each real world outdoor scene [13]. Therefore, in the task of search for threat items in X-ray luggage images it is assumed that stimulus salience is more relevant to naïve observers' attention allocation than experienced screeners since screeners have more experience of targets and the task itself.

In this study, the eye position of screeners and naïve observers was recorded when they searched X-ray airport luggage images for threat items. Data were analysed in terms of performance and visual search factors; including areas of interest attended to, dwell time, and objects selected. The saliency at the first fixation location was extracted from the generated saliency map for each image, by which whether the initial attention location during $X$-ray luggage image searching is determined by visual salience is explored associated with experience of the task. It was hypothesized that naïve observers' attention is more inclined to be guided by stimulus salience than screeners. The differences between screeners and naïve observers are discussed for the development of expertise, visual search skills and of appropriate cognitive schemas.

\section{METHOD}

\section{A. Participants}

Eight students and staff members from Loughborough University and eight airport screeners took part in this study. All participants had normal or corrected-to-normal vision.

\section{B. Stimuli and Apparatus}

Stimuli were $30 \mathrm{X}$-ray air passengers' luggage images. Half of all images were normal bag images. The remaining were target-present images of which each image contained one threat item such as a gun, knife or improvised explosive device (IED). Images were displayed on a 21 inch monitor with a resolution of $1280 \times 1024$ pixels in 32-bit colour mode. Eye movements were recorded by a Tobii eye-tracker (X50) with temporal resolution of $50 \mathrm{~Hz}$ and spatial resolution of $0.35^{\circ}$.

\section{Procedure}

The participants' task was to search X-ray luggage images for threat items of guns, knives or IEDs. At the beginning of the experiment, the eye tracker was calibrated for accurate estimation of an observer's gaze point. The calibration routine displayed a series of calibration points on the screen. Participants were asked to sequentially concentrate on each point. Then participants viewed $30 \mathrm{X}$-ray luggage images in a random order. For each image, decision confidence was rated using a five point scale from 1 'definitely absent' to 5 - 'definitely present'. Other than for decisions of 1 or 2, participants were also asked to indicate the locations of potential threat items. Viewing time was unlimited while participants were required not to spend more time on each image than necessary. Prior to beginning the experiment, for the eight naïve participants, they were introduced to IEDs as being composed of a potential detonator, explosive, wires and a power source connected together. Otherwise, naïve participants had no idea what an IED target would be since they had no knowledge and experience of it.

\section{RESULTS}

\section{A. Performance}

Receiver operating characteristic (ROC) methodology from signal detection theory was used to analyse the decision confidence data using the software ROCKIT [14]. Detection accuracy performance was expressed as $A_{z}$ values, the areas under the ROC curve, which jointly considers hits and misses. Fig. 1 shows the mean ROC 
curves of screeners and naïve observers with sensitivity as the ordinate and 1-specificity as the abscissa. The detection performance of screeners was clearly better than chance while the ROC curve of naîve observers was equivalent to chance. Analysis of independent-samples T-test showed that the performance of screeners was significantly better than the naîve observers, $t=3.77, d f=14, p<0.01$. The overall sensitivity was 0.458 and 0.250 , respectively, for screeners and naïve observers; and specificity was 0.692 and 0.583 , respectively. The difference in sensitivity between naïve observers and screeners was significant, $t=2.913, d f=14, p$ $<0.05$ while the difference in specificity was not significant. The screeners' detection ability was therefore better than the naïve observers.

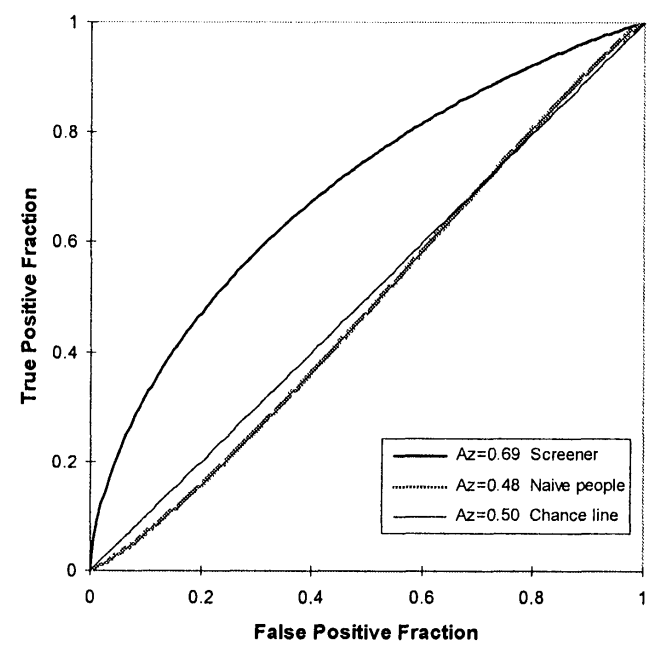

Fig. 1. ROC curves of screener and naïve observers.

To measure task performance, the mean reaction time for accurate target-present and target-absent decisions was calculated. The analysis showed that screeners made decisions much more quickly than the naïve observers, $t=$ 2.475, df $=14, p<0.05$ for accurate target-present responses and, $t=3.913$, df $=14, p<0.01$ for accurate target-absent responses. Fig. 2 shows that the naïve observers took longer than screeners for all responses.

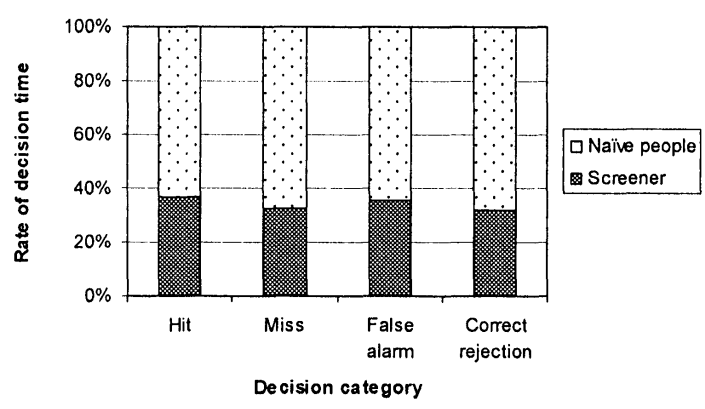

Fig. 2. Rate of decision time between naïve observers and screeners.

\section{B. Visual Search}

Eye position data are recorded by the eye tracker as $X, Y$ coordinates corresponding to the horizontal and vertical dimensions of the displayed image being viewed. Original eye position data are fairly meaningless about how observers are viewing an image unless these are grouped into fixations based on certain spatial and temporal thresholds. Observers process visual information when the eyes fixate on specific areas for a long enough time. In this study, a fixation, or a cluster of fixations, was determined by an algorithm using $2.5^{\circ}$ visual angle as a spatial measure, which is a typical useful field of view size found in medical image inspection research and also in airport luggage image inspection [15], and 200 milliseconds as a minimum fixation duration.

Each target area of a threat item was defined as an Area Of Interest (AOI) for understanding how observers detect or miss targets [16]. Then relative variables, such as; eye position data of the time to first enter the AOI, and the dwell time on the AOI, were calculated. In accord with the decision time of screeners and naïve observers, table 1 shows that the time to first enter the AOI, and also the dwell time on the $\mathrm{AOI}$ of screeners, were shorter than that of the naïve observers. Moreover, the difference between screeners and naïve observers of dwell time on the $A O I$ for the miss responses and the time to first enter the AOI of an IED was significant, $t=3.867, d f=14, p<0.01$ and $t=2.625, d f=14$, $p<0.05$, respectively. The hit rate on an IED for screeners (12/72) was reliably better than naïve observers (31/72) Pearson Chi-Square analysis, $x^{2}=11.97, \mathrm{df}=1, p=0.001$.

TABLE I

STATISTICAL VARIABLES ON AOI OF SCREENER AND NAIIVE OBSERVERS

\begin{tabular}{lllc}
\hline \multicolumn{2}{c}{$\begin{array}{c}\text { Statistic } \\
\text { variables on AOI }\end{array}$} & Screener & $\begin{array}{l}\text { Naive } \\
\text { observers }\end{array}$ \\
\hline Time to & Hit & 1181 & 2342 \\
first & Miss & 1337 & 2389 \\
enter & Gun & 2191 & 2936 \\
AOI & Knife & 1619 & 4951 \\
(msec) & IED & 915 & 1817 \\
\hline Dwell & Hit & 3395 & 4559 \\
time on & Miss & 1475 & 3664 \\
AOI & Gun & 1546 & 3473 \\
(msec) & Knife & 370 & 1009 \\
& IED & 3286 & 4980 \\
\hline \multirow{3}{*}{ Hit rate } & Gun & 0.67 & 0.59 \\
& Knife & 0.33 & 0.17 \\
& IED & 0.43 & 0.17 \\
\cline { 2 - 3 } & Total & 0.46 & 0.25 \\
\hline
\end{tabular}

It was noticed that some targets were not fixated at all while they were still recognized correctly. One of the possible reasons for this is that the dwell time on these target areas was less than the minimum fixation duration in the algorithm. In such a short duration observers were still able to process the visual information and reach a correct judgment. Another possible reason is the effectiveness of peripheral vision which increases the detection of some targets. The hit rate for trials on which fixations fell on target areas following correct responses and hit rate for trials on which no fixations 
TABLE 2

PERCENTAGE OF MISSED THREAT ITMES IN THREE MISS ERROR TYPES FOR NAIIVE PEOPLE AND SCREENERS

\begin{tabular}{ccccccccccc}
\hline & Total & \multicolumn{4}{c}{ Percentage of missed threat items in three miss error types (\%) } \\
\cline { 3 - 10 } Participants & number & \multicolumn{4}{c}{ Search error } & \multicolumn{3}{c}{ Recognition error } & \multicolumn{3}{c}{ Interpretation error } \\
\cline { 3 - 12 } & of miss & Gun & Knife & IED & Gun & Knife & IED & Gun & Knife & IED \\
\hline Naïve people & 91 & 0 & 15.4 & 1.1 & 3.3 & 3.3 & 6.6 & 7.7 & 3.3 & 59.3 \\
Screeners & 65 & 1.5 & 20 & 6.2 & 6.2 & 1.5 & 17 & 4.6 & 3 & 40 \\
\hline
\end{tabular}

fell on target areas following correct responses were calculated for the measurement of target recognition. This analysis found that the hit rate following fixations on targets for screeners was reliably higher than that of the naïve observers, $t=3.046, d f=14, p<0.01$; while there was no difference of hit rate without fixations on targets between the two groups, which both were very low with $17.4 \%$ and $11.8 \%$, respectively. Once threat item targets were fixated, screeners were able to recognise them more with their expertise and experience than the naïve observers. If the threat item targets were not fixated then both screeners and naïve observers had a similar hit rate. The false alarm rate of naïve observers was 0.41 , which was higher than that for the screeners of 0.30 . All of the information indicated that some threat items were recognized by chance when naïve observers made a decision.

Miss errors were classified into three types based on the useful field of view and gaze duration [17]. In this study, AOI was taken as a reference for the target area so that if observers fixated on the AOI, then targets were scored as hits by the eye fixations. Then if targets are not hit by any fixations, such a miss error is called a search error due to inadequate visual attention. If targets are hit by fixations and the gaze duration is less than $1000 \mathrm{~ms}$, then the miss error is called a recognition error. Furthermore, if targets are fixated for more than $1000 \mathrm{~ms}$, then this is termed an interpretation error. Most of the threat items were missed due to interpretation errors for both the naivve observers and the screeners whilst the percentage of interpretation error of the naïve observers was higher than that of the screeners (see table 2). Although participants fixated on target areas for a longer period and processed the visual information, they still missed the targets due to their lack of knowledge of what constituted such a threat item. Missed IEDs accounted for the main percentage of interpretation errors while most of the knives were missed because of search errors which was one of the possible reasons that the dwell time on knives was the shortest, compared with guns and IEDs.

\section{Initial Visual Selective Attention}

Performance and eye movement data analysis showed that the screeners' advantages were of speed and accuracy when they searched for threat items in X-ray luggage images. In addition, screeners were faster to fixate on target areas than naïve observers, especially for IEDs. Is there any difference in the initial visual attention paid to $X$-ray luggage images between screeners and naïve observers? The salience value of the first fixation point of the screeners and naïve observers was extracted from the corresponding saliency map to examine whether there was a difference of stimulus properties of these initial attentive locations. If salience at the first fixation location of naîve observers is greater than that of screeners, then this may indicate that naîve observers are more likely attracted by salient regions at the first fixation.

Itti and Koch's biologically pure model of bottom-up visual selective attention predicts where the observer fixates in a complex scene on the basis of salient areas which are determined by complicated processing and algorithm. The saliency model of Itti and Koch was used to generate a saliency map [http://www.saliencytoolbox.net/download.html, retrieved on 21, December, 2006]. Fig. 3 is an example of an original $X$-ray luggage image [http://www. delftoutlook.tudelft.nl/info/images/ACF1381.jpg, retrieved on 13, July, 2007], and its generated saliency map with regions of high salience shown in white. To obtain the saliency map, low level vision features (colour, intensity and orientation) are extracted from the input of the colour image at nine spatial scales from $1: 2^{0}$ (level 0 ) to $1: 2^{8}$ (level 8 ) by a Gaussian pyramid scheme. After the linear filtering visual features are processed by centre-surround differences to maximize local differences and increase spatial contrast in each feature channel to obtain a total of 42 feature maps. Then multiple feature maps are normalized which eliminates feature-dependent factors by two-dimensional difference-ofGaussians filter. The program ('saliencytoolbox') adopts the method of "max-normalize" which maximizes the local difference so that the real salience can not be found from the saliency map. For computational convenience a change was performed for normalization such that salience was scaled to range from 0 to 255. After normalization, three conspicuity maps for colour, intensity and orientation are obtained and linearly summed into the unique saliency map.

For each of the $30 \mathrm{X}$-ray luggage images a visual saliency map was calculated. Then the mean salience at the first fixation location of each participant for all images was found. The salience at the first fixation location of screeners and naïve observers was 137 and 140, respectively. The difference was not significant, $t(14)<1$.
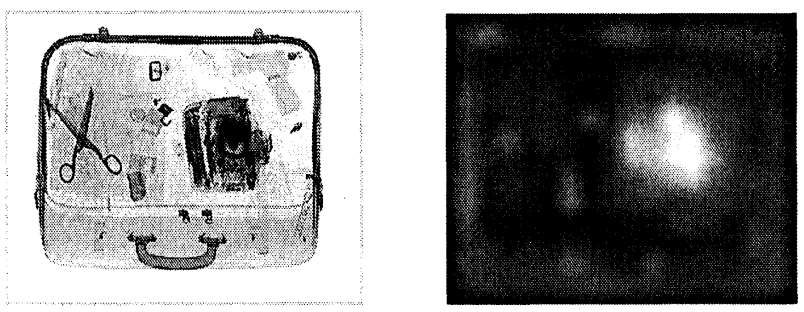

Fig. 3. Example of an X-ray luggage image (left) and its generated saliency map (right). 


\section{DISCUSSION}

In this study, the difference between naïve observers and screeners on performance, visual scanning and recognition skills was explored when they completed a simulated airport security examination task. As expected, screeners detected and recognized threat items such as guns, knives and IEDs more quickly and accurately than naïve observers. Better detection ability of screeners was shown by significantly higher sensitivity and a slightly lower false alarm rate than naïve observers. Also, the visual search of screeners was more effective with a shorter time to fixate on target areas than naïve observers, especially for IEDs. It is impossible for observers to learn about IED appearances in everyday life so screeners benefitted from training and experience in recognising IEDs. However, the hit rate on IEDs of the screeners was not high in this study. Analysis of the reason for errors showed that most of the IEDs were missed due to interpretation errors where participants fixated on target areas for a long time but they still missed them. This indicated that as the form of IED is very changeable so that it is necessary for screeners to update their knowledge of such threat item appearances. Search errors were the main miss error for knives in this study. One possible reason was that these targets are relatively small within a cluttered luggage background. Generally, screeners detect knives with the assistance of the displayed image using colour to indicate a metal present in the luggage item, e.g. inorganic materials are marked by blue. If knives are made with any low-density materials such as wood, glass or epoxy fibre, then it is possible for an observer to ignore them - regular and appropriate training is a solution for this problem.

It was interesting that participants detected some targets even when they did not fixate on them. Although the hit rate following no fixation on a target was very low for naïve observers and screeners, it suggested the effectiveness of peripheral vision within visual search. Search and decisionmaking, consecutive processes, were separated into three stages in this study: search - decision confidence rating and indicating a potential threat location. Therefore, it was possible that participants continued processing uncertain and suspected visual information which was obtained via peripheral vision during the previous visual fixation when they indicated the locations of potential threats. This could be examined by further subtle experimental investigations.

There was no difference of salience at the first fixation locations between naïve observers and the screeners, which indicated that locations with similar salience were selected by both naïve and experienced observers in the initial global stage of search for threat items in X-ray air passenger luggage images. Salience, as a quantitative index, does not distinguish between novice and expert at the beginning of their search stage. However, which selective attention mechanism, bottom-up or top-down, plays the dominant role in luggage image examination and how visual salience influences eye positions with the course of search is not addressed from the results. To answer the questions, the fixation number of human observers in a salient region is compared with the number of fixations generated by a random model in a planned future study. If the fixations of the human observers in salient regions are more than the random model, then the saliency map can predict human eye movements. Otherwise, the stimulus-driven element does not contribute significantly to selective attention in the task of luggage image inspection. Also the difference between the salience of human fixations and the salience expected by the saliency map model is tracked with the course of visual search for exploring stimulus dependence with exposure time. If all this is achieved, then not only will a better training scheme be provided but assistant systems with automatic predictions of salient locations can then be embedded into an advanced airport security scan systems as a perceptual support.

\section{CONCLUSIONS}

The advantages of screeners are speed and accuracy in the task of searching for threat items in air passengers' $X$-ray luggage images. Their visual search was not only efficient but also effective. However, screeners still missed IED targets, although they had fixated on them which indicated that screeners should regularly update knowledge of threat item appearances frequently. There was no difference in salience at the first fixation locations between naîve observers and screeners. The saliency map model could not distinguish between naïve and experienced observers at the global search stage. In other words, experience of such X-ray luggage images and the inspection task did not change the visual attention preference of stimuli property at the early stage of search for the experienced observers. More work is clearly needed to understand the visual selective attention mechanism for building a saliency assistant system in airport security screening.

\section{ACKNOWLEDGEMENTS}

This research is supported by the EPSRC and carried out in collaboration with QinetiQ.

\section{REFERENCES}

[1] D. H. Harris, "How to Really Improve Airport Security," Ergonomics in Design, vol. 10, pp. 17-22, 2002.

[2] J. E. Hoffman, "A two-stage model of visual search," Perception \& Psychophysics, vol. 25, pp. 319-327, 1979.

[3] R. G. Swensson, "A Two-stage Detection Model Applied to Skilled Visual Search by Radiologists," Perception \& Psychophysics, vol. 27, pp. 11-16, 1980.

[4] A. G. Gale, "Human Response to Visual Stimuli," in W. Hendee and P. Wells. Eds., The Perception of Visual Information. New York, Springer Verlag, 1997, pp. 127147.

[5] H. E. Egeth and S. Yantis, "Visual attention: Control, Representation, and Time Course," Annual Review Psychology, 1997, vol. 48, pp. 269-297.

[6] H. L. Kundel, C. F. Nodine, D. Thickman and L. Toto, "Searching for lung nodules: A comparison of human performance with random and systematic scanning models," Investigative Radiology, vol. 22, pp. 417-422, 1987.

[7] H. L. Kundel and P. S. LaFollette, "Visual Search Patterns and Experience with Radiological Images," Radiology, vol. 103, pp. 523-528, 1972. 
[8] E. A. Krupinski, "Influence of Experience on Scanning Strategies in Mammography," in H. L. Kundel, Ed., Medical Imaging: Imaging Perception, Proceeding of SPIE, 1996, vol. 2712, pp. 89-94.

[9] C. F. Nodine, H. L. Kundel, C. Mello-Thoms, S. P. Weinstein, S. G. Orel, D. C. Sullivan, and E. F. Conant, "How Experience and Training Influence Mammography Expertise," Academic Radiology, vol. 6, pp. 575-585, 1999.

[10] L. Itti and C. Koch, "Computational Modelling of Visual Attention," Nature Reviews Neuroscience, vol. 2, pp. 194-203, 2001

[11] L. Itti and C. Koch, "A Saliency-Based Search Mechanism for Overt and Covert Shifts of Visual Attention," Vision Research, vol. 40, pp. 1489-1506, 2000.

[12] D. Parkhurst, K. Law and E. Niebur, "Modeling the Role of Salience in the Allocation of Overt Visual Attention," Vision Research, vol. 42, pp. 107-123, 2002.

[13] J. M. Henderson, J. R. Brockmole, M.S. Castelhano and M. Mack, "Visual Saliency does not Account for Eye Movements during Visual Search in Real-World Scenes," in R. Van Gompel, M. Fischer, W. Murray and R. Hill, Eds., Eye Movements: A Window on Mind and Brain. Oxford: Elsevier, 2007, pp. 537-562.

[14] C. E. Metz, B. A. Herman and J-H. Shen, "MaximumLikelihood Estimation of ROC Curve from ContinuouslyDistributed Data," Statistics in Medicine, vol. 17, pp. 1033-1053, 1998.

[15] A. G. Gale, M. Mugglestone, K. J. Purdy and A. McClumpha, "Is airport baggage inspection just another medical image?" In: E.A. Krupinski, Ed., Image Perception and Performance, Proceeding of SPIE, 2000, vol. 3981, pp. 184-192.

[16] X. Liu, A. G. Gale, K. Purdy and T. Song, "Is that a gun? The influence of features of bags and threat items on detection performance," In: P. D. Bust, Ed., Contemporary Ergonomics, London: Taylor and Francis, 2006, pp. 17-22.

[17] H. L. Kundel, C. F. Nodine and D.Carmody, "Visual scanning, pattern recognition and decision-making in pulmonary nodule detection," Investigative Radiology, vol. 13, pp. 175-181, 1978.

\section{VITA}

Xi Liu received the BSc. and MSc. degrees from Tsinghua University, China, and Chinese Academy of Sciences, in 2000 and 2003 respectively. Now she is a Ph.D. candidate in Applied Vision Research Centre of Loughborough University. Her research interests include applied visual search, attention mechanisms and human performance.

Alastair Gale graduated from the University of Durham, England with a BSc degree in psychology and a PhD on the role of eye movements in figure perception. He is a Fellow of the British Psychological Society, a Chartered Psychologist, a Chartered Health Psychologist, a Registered Ergonomist, and a Fellow of the Ergonomics Society. He is also an honorary member of the Royal College of Radiologists' Breast Group and was awarded honorary membership of the Royal College of Radiologists in 1998 for his radiological research. Alastair has been Professor of Applied Vision Sciences at Loughborough University, England since 2005.

Tao Song received his BSc. in Computer Science from Zhong Shan University, China in 1995. He got his MSc. in Colour Imaging and successfully completed his $\mathrm{PhD}$ from Derby University, UK, in 1999 and 2007 respectively. His research interests include Human Vision, Computer Vision, computer and imaging technologies. 